Article

\title{
Social Network Analysis of Twitter Data Related to Blood Clots and Vaccines
}

\author{
Wasim Ahmed ${ }^{1}$, Josep Vidal-Alaball2,3,4 *, Josep M Vilaseca ${ }^{4}$ \\ 1 Stirling University Management School, University of Stirling; wasim.ahmed@stir.ac.uk \\ 2 Health Promotion in Rural Areas Research Group, Gerència Territorial de la Catalunya Central, Institut \\ Català de la Salut; jvidal.cc.ics@gencat.cat \\ 3 Unitat de Suport a la Recerca de la Catalunya Central, Fundació Institut Universitari per a la recerca a l'A- \\ tenció Primària de Salut Jordi Gol i Gurina \\ 4 Faculty of Medicine. University of Vic - Central University of Catalonia josepmaria.vilaseca@umedicina.cat \\ * Correspondence: jvidal.cc.ics@gencat.cat; Tel.: +936930040
}

\begin{abstract}
After the first weeks of vaccination against the SARS-CoV-2, several cases of acute thrombosis were reported. These news reports began to be shared frequently across social media platforms. The aim of this study was to conduct an analysis of Twitter data related to the overall discussion. Data was retrieved from 14th March to 14th April using the keyword 'blood clots'. A dataset with $n=266,677$ tweets was retrieved, and a systematic random sample of $5 \%$ of tweets $(n=13,334)$ were entered into NodeXL for further analysis. Social network analysis was used to analyse the data by drawing upon the Clauset-Newman-Moore algorithm. Influential users were identified by drawing upon the betweenness centrality metric. Text analysis was applied to identify the key hashtags and websites used at this time. More than half of the network was comprised of retweets and the largest groups within the network were broadcast clusters where a number of key users were retweeted. The most popular narratives were around highlighting the low risk of obtaining a blood clot from a vaccine and highlighting higher blood clot risks in medicines commonly consumed. A wide-variety of actors drove the discussion on Twitter ranging from writers, physicians, the general public academics, celebrities, and journalists. Twitter was used to highlight the low potential of obtaining a blood clot from a vaccine and encouraged vaccinations among the public.
\end{abstract}

Keywords: COVID-19; Twitter; Blood Clots; Social Media; Clots

\section{Introduction}

Since the beginning of the coronavirus disease 2019 (COVID-19) pandemic, caused by the SARS-CoV-2 virus in 2019 [1] it has become apparent that one of the features of COVID-19 is thrombosis (popularly known as blood clots) [2]. Several studies confirmed the observation made by initial publications on thrombotic complications in COVID-19 patients that a diagnosis of thromboembolism per se was associated with a more complicated in-hospital clinical course, higher incidence of admittance to the intensive care unit and higher all-cause mortality [3]. However, the early identification of patients at risk of thrombosis is still a challenge. Protocols to treat COVID-19 may differ across Europe. The role of heparin in the prevention of thrombosis and acute embolism seems of importance in severely ill COVID-19 patients [4]. In Spain, hospitalised patients are usually treated with heparin, whereas homecare patients are not.

After the first weeks of vaccination against the SARS-CoV-2, several cases of acute thrombosis were reported. Those cases were mainly associated with the AZD1222 (Oxford-AstraZeneca) and Janssen COVID-19 (Johnson \& Johnson) vaccines. The events had a great impact on the media, and a debate emerged on the safety of COVID-19 vaccines in 
general. The 10th of March 2021, Austria had suspended the use of a batch of AstraZeneca vaccines after one person had multiple thrombosis diagnosed and died 10 days after vaccination [5]. The day after, eight European countries suspended the use of AstraZeneca's vaccine [6]. Italy, France, Germany, and Spain suspended use of the vaccine on the 15th of March. This cascade of European countries ceasing the vaccination programme prompted the pharmacovigilance agencies to react. Hence, on the 18th of March, both the British Medicine and Healthcare Regulatory Authority (MHRA) and the European Medicines Agency (EMA) stated independently that the AstraZeneca vaccine is safe, effective, and that there is no evidence of association between the vaccine and the notified cases of blood clots [7].

The national authorities did not feel fully satisfied with these statements. On the 25th of March the EMA published a note saying that "the committee confirmed that the vaccine is not associated with an increase in the overall risk of blood clots and that the benefits of the vaccine in combating the still widespread threat of COVID-19 continue to outweigh the risk of side effects". The committee recommended including more information and advice for healthcare professionals and the public in the vaccine's product information [8]. Immediately, AstraZeneca modified the product's information and published a direct communication to healthcare professionals warning of the notified cases of thrombosis after vaccination and reminding the signs and symptoms of thrombosis [9]. The text is ambiguous when describing the relationship between vaccination and blood clots, and the conclusion is unclear. Whatever the relationship between the vaccine and thrombosis, the European Commission decided not to buy the vaccine from 14th of April $2021[10]$.

Governments and public health authorities have noted the potential of large-scale vaccination programmes as a way out of the COVID-19 Pandemic. Reports of blood clots may potentially deter certain individuals from obtaining a vaccine and can put in danger the aim to control the pandemic. Although vaccination has been shown throughout history to have great benefits, vaccine hesitancy can be a major public health problem and WHO has considered it one of the top ten threats to global health $[11,12]$.

It is well known that with the eclosion of social media, anti-vaccine movements have actively used them in order to spread rumours about the alleged dangers of vaccines [13], Since the advent of COVID-19, discussions around COVID vaccines have been taking place on social media [14], and lately, discussions around blood clots have been discussed frequently on Twitter. From a public health standpoint, it is important to gain an understanding of the types of discussions taking place. The aim of this study is to analyse Twitter discussions around thrombosis related events associated with COVID-19 vaccination. Our results are likely to be of interest to health organisation and governments around the world.

\section{Materials and Methods}

This study retrieved data from the Twitter Search Application Programming Interface (API) using the Twitter Archiving Google Sheets (TAGS). This API is likely to provides enough tweets if the time-duration is short, and there are few keywords used which was the case in our study. Previous research has found that for a single keyword search, the Search API may be able to retrieve up to $79 \%$ of all tweets when compared to the Firehose (which provides all tweets) [15]. Data was retrieved from 14th March to 14th April using the keyword 'blood clots'. A dataset with $n=266,677$ tweets was retrieved, and a systematic random sample of $5 \%$ of tweets $(n=13,334)$ were entered into NodeXL for further analysis. We used a similar methodology to previous research [16,17]. Social network analysis was used to analyse the data which is useful in highlighting the key groups of discussion within the network. A network graph was produced by analysing relationships between Twitter accounts such as tweets, retweets, and mentions and ac- 
counts that conversed among each other were assigned into distinct groups within the network. More specifically, the Clauset-Newman-Moore algorithm was used to identify the key communities within the discussion. Influential users were identified by using the betweenness centrality algorithm. The study fell under a larger project that has full ethical approval. The study made use of only public data which was analysed in aggregate.

\section{Results}

There were 13,874 Twitter users within our dataset. The dataset was comprised of $\mathrm{n}=9,900$ retweets, $\mathrm{n}=1,952$ tweets, $\mathrm{n}=1293$ replies, $\mathrm{n}=1261$ mentions, and $\mathrm{n}=765$ mentions in retweet. This highlights that the network had a high retweet ratio and was a broadcast network. The social network analysis of the data revealed that the discussion around blood clots was formed across several communities. Figure 1 is a diagrammatic representation of Twitter users. Each circle represents Twitter users, and the larger circles represent users who were influential and smaller dots represent users that were less influential within the network. The groups are presented from left to right by size. The four largest groups have been labelled (Group 1, Group 2, Group 3, and Group 4).

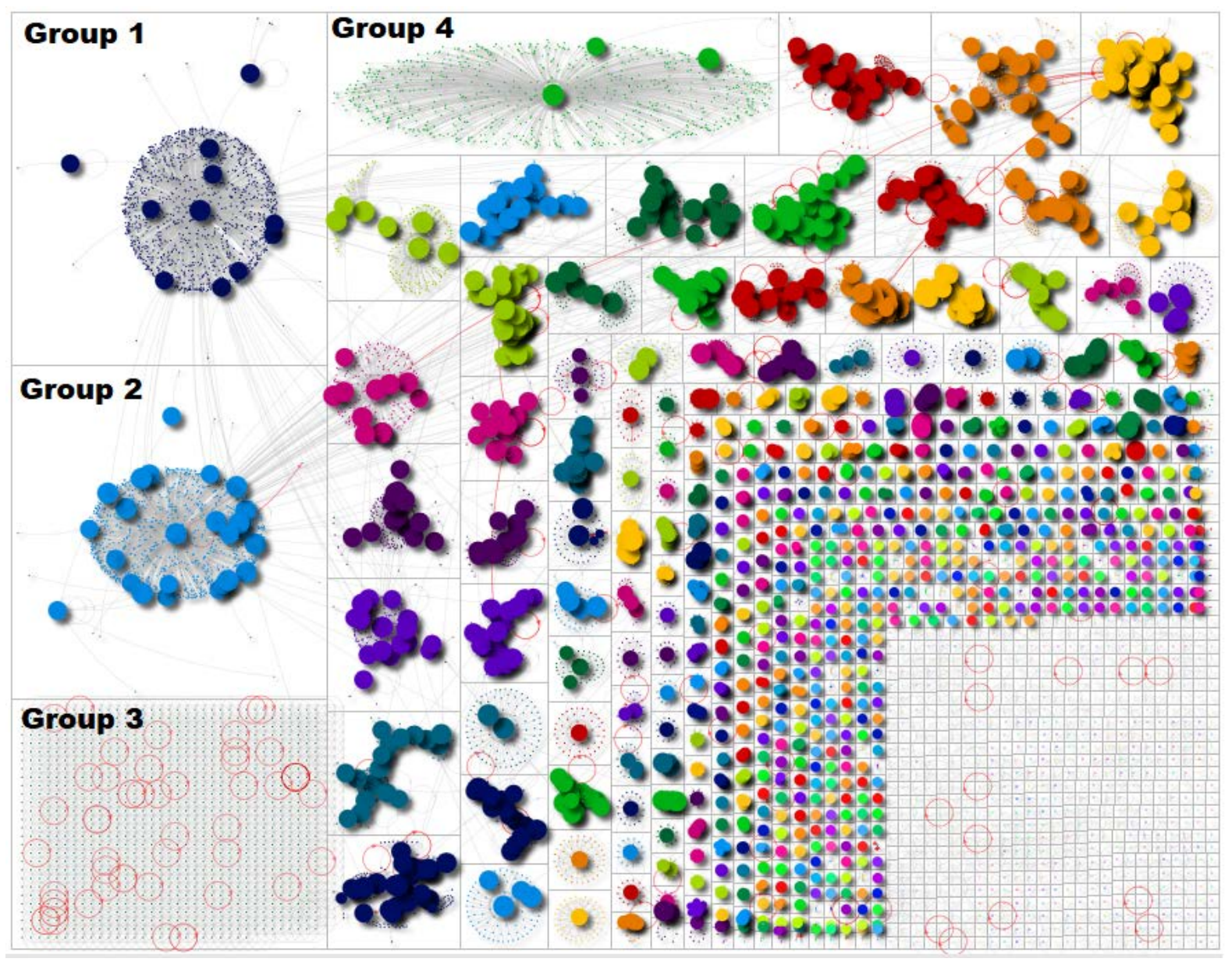

Figure 1. Social Network Analysis of the keyword 'blood clots' from 14th March to 14th April 2021

The figure highlights how there were four key groups of users followed by a number of smaller pockets of discussion. Groups 1, 2 and 4, contained tweets downplaying the risk of obtaining a blood clot from a vaccine and these tweets were amplified within these broadcast groups.

Table 1 below provides an overview of the key websites used during this time. The most retweeted tweets in each of the two largest clusters were identified.

In group 1 the most retweeted tweet was as follows: 
"So the odds of getting a blood clot from the JEJ vaccine are literally one in a million and they stopped administering it. Do you know the odds of getting blood clots from birth control? It's 1/1000. (This is considered very low odds)"

The most retweeted tweet in group 2 was as follows:

"3 key points for folks recently vaccinated with JEJ

1. your risk of getting hit by lightning this year > your risk of developing blood clot from vaccine

2. Don't ignore a new severe headache, abd pain, or shortness of breath

3. Enjoy being vaccinated against a deadly disease"

Table 1. Overview of key websites

\begin{tabular}{|c|c|c|}
\hline Title & $\begin{array}{c}\text { Top URLs in Tweet in Entire } \\
\text { Graph }\end{array}$ & No. of times shared \\
\hline $\begin{array}{l} \\
\text { CDC, FDA To Review J\&J Shot } \\
\text { After } 6 \text { Blood Clot Cases Re- } \\
\text { ported Out Of Nearly 7M Doses }\end{array}$ & $\begin{array}{l}\frac{\text { https://www.npr.org/sections/co }}{\frac{\text { rona- }}{\text { vi- }}} \\
\frac{\text { rus-live-updates/2021/04/13/9867 }}{\text { 09618/u-s-recommends-pausing- }} \\
\frac{\text { use-of-johnson-johnson-vaccine- }}{\text { over-blood-clot-concerns?utm s }} \\
\frac{\text { ource=twitter.com\&utm mediu }}{\text { m=social\&utm term=nprnews\& }} \\
\underline{\text { utm campaign=npr }}\end{array}$ & 222 \\
\hline $\begin{array}{l}\text { He won't allow over-the-counter } \\
\text { birth control, but Biden is push- } \\
\text { ing risky at-home abortions }\end{array}$ & $\begin{array}{l}\text { https://www.washingtonexamin } \\
\text { rer.com/opinion/he-wont-allow-o } \\
\begin{array}{c}\text { ver-the-counter-birth-control-bu } \\
\text { t-biden-is-pushing-risky-at-hom } \\
\text { e-abortions }\end{array} \\
\end{array}$ & 93 \\
\hline $\begin{array}{l}\text { If you've recently had the J\&J } \\
\text { vaccine, watch for these rare } \\
\text { symptoms, CDC says }\end{array}$ & https://trib.al/RfZg4Ut & 83 \\
\hline Link to USA FDA Tweet & $\begin{array}{c}\text { https://twitter.com/US FDA/stat } \\
\underline{\text { us/1381925612743499778 }}\end{array}$ & 82 \\
\hline $\begin{array}{l}\text { Blood clot risks: comparing the } \\
\text { AstraZeneca vaccine and the } \\
\text { contraceptive pill }\end{array}$ & $\begin{array}{l}\frac{\text { https://theconversation.com/blo }}{\text { od-clot-risks-comparing-the-astr }} \\
\frac{\text { azene- }}{\text { ca-vaccine-and-the-contraceptiv }} \\
\text { e-pill-158652 }\end{array}$ & 82 \\
\hline $\begin{array}{c}\text { Clot questions over AstraZeneca } \\
\text { and J\&J vaccine }\end{array}$ & $\begin{array}{l}\text { https://news.yahoo.com/clot-que } \\
\underline{\text { stions-over-astrazeneca-j-204253 }} \\
\underline{695 . h t m l}\end{array}$ & 75 \\
\hline $\begin{array}{l}\text { US calls for pause in Johnson \& } \\
\text { Johnson vaccinations over blood } \\
\text { clot concerns }\end{array}$ & https://trib.al/Er6CJdw & 65 \\
\hline $\begin{array}{l}\text { CDC, FDA To Review J\&J Shot } \\
\text { After } 6 \text { Blood Clot Cases Re- } \\
\text { ported Out Of Nearly 7M Doses }\end{array}$ & $\begin{array}{l}\frac{\text { https://choice.npr.org/index.htm }}{\text { l?origin=https://www.npr.org/se }} \\
\frac{\underline{\text { ctions/coronavirus-live-updates/ }}}{\frac{2021 / 04 / 13 / 986709618 / \text { u-s-recom }}{\text { mends-pausing-use-of-johnson-j }}} \\
\frac{\text { ohn- }}{\underline{\text { son-vaccine-over-blood-clot-con }}} \\
\frac{\text { cerns }}{n}\end{array}$ & 57 \\
\hline
\end{tabular}




\begin{tabular}{|c|c|c|}
\hline $\begin{array}{l}\text { CDC and FDA recommend US } \\
\text { pause use of Johnson \& John- } \\
\text { son's Covid-19 vaccine over } \\
\text { blood clot concerns }\end{array}$ & $\frac{\frac{\text { https://www.cnn.com/2021/04/1 }}{\text { 3/health/johnson-vaccine-pause- }}}{\text { cdc-fda/index.html }}$ & 49 \\
\hline $\begin{array}{l}\text { Canadian public health agency } \\
\text { confirms first report of blood } \\
\text { clot linked to AstraZeneca }\end{array}$ & $\begin{array}{l}\frac{\text { https://nationalpost.com/news/c }}{\underline{\text { anadi- }}} \\
\frac{\text { an-press-newsalert-phac-receive }}{\text { s-report-of-blood-clot-linked-to- }} \\
\underline{\text { astrazene- }} \\
\frac{\text { ca?utm medium=Social\&utm s }}{\text { ource=Twitter\#Echobox }=1618336} \\
\underline{916}\end{array}$ & 42 \\
\hline
\end{tabular}

It can be seen that a range of websites were shared reporting on general news from the time-period that was examined. These web-sources were mainly based on news updates reporting on the links between blood clots and vaccines from around the world such as the United States and Canada.

Table 2 below provides an overview of the most used hashtags.

Table 2. Overview of key hashtags

\begin{tabular}{cc}
\hline Hashtag & No. of times used \\
\hline johnsonandjohnson & 260 \\
covid19 & 195 \\
vaccine & 118 \\
astrazeneca & 85 \\
breaking & 53 \\
covidvaccine & 33 \\
coronavirus & 33 \\
covid & 25 \\
covid19ontario & 24 \\
wsjwhatsnow & 18 \\
\hline
\end{tabular}

The five most used hashtags (side from covid19) were \#johnsonandjohnson $(\mathrm{n}=260)$, \#vaccine $(\mathrm{n}=118)$, \#astrazeneca $(\mathrm{n}=85)$, \#breaking $(\mathrm{n}=53)$, and \#covidvaccine $(\mathrm{n}=33)$. Overall, hashtags were used to have discussions around blood clots reported in certain vaccines. It is not surprising to see 'johnsonandjohnson' and 'astrazeneca' appear among the most used hashtags because news reports and discussions were frequently mentioning them.

Table 3 provides an overview of the most utilised word-pairs.

Table 3. Overview of word-pairs

\begin{tabular}{cc}
\hline Top Word Pairs in Tweet in Entire Graph & Entire Graph Cont \\
\hline blood,clot & 13709 \\
birth,control & 3581 \\
clot,vaccine & 3503 \\
odds,blood & 2911 \\
blood,clots & 2620 \\
chance,blood & 2030 \\
johnson,johnson & 1798 \\
250,000, chance & 1764 \\
control,1000 & 1491 \\
Developing,blood & 1435 \\
\hline
\end{tabular}

As shown in Table 3 above, Twitter users conversed about blood clots in relation to the vaccine and co-words such as 'blood, clot', 'clot, vaccine', 'blood, clots' and 'johnson, johnson' appeared. These discussions were related to the general situation. A further 
group of keywords discussed the odds of getting a blood clot and utilised keywords such as 'odds,blood', 'chance, blood', ' 1 in 250,000, chance', and 'control, 1 in 1000', and 'developing, blood'. Twitter users also discussed the higher chance of getting a blood clot from birth control and the word pair 'birth, control' appeared.

Table 4 below provides an overview of the types of users that were driving the discussion on Twitter.

Table 4. Overview of Influential Users within the network

\begin{tabular}{cc}
\hline Rank & Userhandle \\
\hline 1 & Writer \\
2 & Physician \\
3 & Member of Public \\
4 & US Food and Drug Administration \\
5 & Member of Public \\
6 & National Public Radio (United States) \\
7 & Academic \\
8 & Cable News Network (CNN) \\
9 & Celebrity \\
10 & Journalist \\
\hline
\end{tabular}

A number of influential users across disciplines ranging from writers, physicians, the general public, academics, celebrities, and journalists were influential in driving the key narratives and popular messages that were highlighted in Table 4.

\section{Discussion}

Social media platforms are a source of health information for some members of the public and can have considerable influence on health decision making. Effective routes out of the current pandemic include large-scale vaccinations and public confidence in vaccines plays an important role on uptake. News of vaccines potentially causing blood clots became a major news story and was discussed frequently on Twitter. To the best of our knowledge, this is the first article analysing the discussions that have taken place on social media around COVID-19 vaccination and specifically the associated risk of thrombosis. Our study sought to develop an understanding of the key narratives, groups, and content within the network. It was found that the network had a high retweet ratio and resembled a broadcast network with high amplification. The two most popular tweets within group 1 and 2 served to encourage vaccinations and appeared to downplay the risk of blood clot events occurring. These discussions were driven by influencers across a broad range of disciplines. In our globalised world, social networks debates may influence the behaviour of individuals and communities. An interesting finding was that the majority of influential users and key websites were not from the world of scientific medicine. Instead, it was found that the majority of information is provided by non-medical sources. An implication of this is that the general scientific studies such as peer-reviewed papers and other scientific health information is being converted into easily consumable content by the general public, academics, celebrities, and journalists. Our study highlights how such stakeholders were playing a positive role in sharing factual information. This is because the largest groups within the network shared content that downplayed the risk of obtaining a blood clot and encouraged users to get their vaccines. The response was community driven and decentralised.

However, what if this broadcast was discouraging people to get vaccinated? What if there would be a negative impact in the vaccination uptake? The misuse of powerful social networks can be a danger for public health campaigns to be effective. Even more, 
how can policy makers become key influencers in the networks in order to enhance public health campaigns?

In our study we found that social networks were reinforcing a global public health vaccination campaign. However, the aim could have been the opposite. We suggest healthcare stakeholders to be involved in the communication of scientific evidence in the social media. Our results show an unintended positive message in favour of COVID-19 vaccination driven by non - healthcare influencers. The challenge to healthcare stakeholders is becoming part of this network. As a study suggests [18], for COVID-19, social media can have a crucial role in disseminating health information and tackling infodemics and misinformation.

One of the limitations of our study is that we were unable to relate the tweets and retweets with their geographical area. If it were possible, we could relate the social network activity in a certain geographical area with the coverage of vaccination in this area during a certain period. However, our results are likely to represent content that was popularly being read and share in English speaking countries because our keyword to retrieve data was in English and because the United States has the most Twitter users. We encourage researchers to go forward on this line of research to understand the effectiveness of the messages amplified by the social networks by specific regions. However, our present results are likely to be of interests to stakeholders working in this area.

\section{Conclusions}

Twitter was used to highlight the low potential of obtaining a blood clot from a vaccine and encouraged vaccinations among the public. Our results are likely to be of interest to health authorities, governments and stakeholders involved in vaccination programmes.

Author Contributions: For research articles with several authors, a short paragraph specifying their individual contributions must be provided. The following statements should be used "Conceptualization, X.X. and Y.Y.; methodology, X.X.; software, X.X.; validation, X.X., Y.Y. and Z.Z.; formal analysis, X.X.; investigation, X.X.; resources, X.X.; data curation, X.X.; writing-original draft preparation, X.X.; writing-review and editing, X.X.; visualization, X.X.; supervision, X.X.; project administration, X.X.; funding acquisition, Y.Y. All authors have read and agreed to the published version of the manuscript." Please turn to the CRediT taxonomy for the term explanation. Authorship must be limited to those who have contributed substantially to the work reported.

Funding: This research received no external funding.

Conflicts of Interest: The authors declare no conflict of interest.

\section{References}

1. Wu F, Zhao S, Yu B, Chen Y, Wang W, Song Z, et al. A new coronavirus associated with human respiratory disease in China. Nature 2020 Feb 3;579(7798):265-269.

2. Levi M, Thachil J, Iba T, Levy JH. Coagulation abnormalities and thrombosis in patients with COVID-19. Lancet Haematol. 2020 Jun;7(6):e438-e440. doi: 10.1016/S2352-3026(20)30145-9. Epub 2020 May 11. PMID: 32407672; PMCID: PMC7213964.

3. Barco S, Bates SM, Versteeg HH, Klok FA. COVID-19 in thrombosis research: An editorial perspective. Thromb Res. 2021 Mar 24;201:147-150. doi: 10.1016/j.thromres.2021.03.011. Epub ahead of print. PMID: 33798826; PMCID: PMC7987507.

4. Stasi C, Fallani S, Voller F, Silvestri C. Treatment for COVID-19: An overview. Eur J Pharmacol. 2020 Dec 15;889:173644. doi: 10.1016/j.ejphar.2020.173644. Epub 2020 Oct 11. PMID: 33053381; PMCID: PMC7548059.

5. COVID-19 Vaccine AstraZeneca: PRAC preliminary view suggests no specific issue with batch used in Austria: https://www.ema.europa.eu/en/news/covid-19-vaccine-astrazeneca-prac-preliminary-view-suggests-no-specific-issue-batc h-used-austria [last visit 17th December 2021].

6. Wise J. Covid-19: European countries suspend use of Oxford-AstraZeneca vaccine after reports of blood clots. BMJ. 2021 Mar 11;372:n699. doi: 10.1136/bmj.n699. PMID: 33707182.

7. Merchant HA. CoViD vaccines and thrombotic events: EMA issued warning to patients and healthcare professionals. J Pharm Policy Pract. 2021 Mar 24;14(1):32. doi: 10.1186/s40545-021-00315-w. PMID: 33761987; PMCID: PMC7988638. 
8. EMA's safety committee continues investigation of COVID-19 Vaccine AstraZeneca and thromboembolic events - further update:

https://www.ema.europa.eu/en/news/emas-safety-committee-continues-investigation-covid-19-vaccine-astrazeneca-throm boembolic-events [last visited 17th December 2021].

9. COVID-19 Vaccine AstraZeneca: Risk of thrombocytopenia and coagulation disorders: https://www.ema.europa.eu/en/documents/dhpc/direct-healthcare-professional-communication-dhpc-vaxzevria-previous ly-covid-19-vaccine-astrazeneca en.pdf [last visited 17th December 2021]

10. European Union opts not to renew AstraZeneca vaccines contract after supply row I Euronews [Internet]. euronews. 2021 $\begin{array}{lllll}\text { [cited } 2021 & \text { May } & \text { Available from: }\end{array}$ https://www.euronews.com/2021/05/09/european-union-opts-not-to-renew-astrazeneca-vaccines-contract-after-supply-ro $\underline{\mathrm{W}}$

11. World Health Organization. Ten threats to global health in 2019 [Internet]. 2019 [cited 2021 May 16]. Available from: https://www.who.int/news-room/spotlight/ten-threats-to-global-health-in-2019 2.

12. Salmon DA, Dudley MZ, Glanz JM, Omer SB. Vaccine Hesitancy: Causes, Consequences, and a Call to Action. Am J Prev Med. 2015 Nov 1;49(6):S391-8.1.

13. Gunaratne K, Coomes EA, Haghbayan H. Temporal trends in anti-vaccine discourse on Twitter. Vaccine. 2019 Aug 14;37(35):4867-71.

14. Puri N, Coomes EA, Haghbayan H, Gunaratne K. Social media and vaccine hesitancy: new updates for the era of COVID-19 and globalized infectious diseases. Hum Vaccines Immunother. 2020;16(11):1-8.

15. Ahmed, W. and Bath, P.A. (2015) Comparison of Twitter APIs and tools for analysing Tweets related to the Ebola Virus Disease. In: iFutures 2015. iFutures: iSchool Postgraduate Research Conference, 7 July 2015, University of Sheffield, Sheffield. iFutures. Sheffield iSchool Conference

16. Ahmed W, Marin-Gomez X, Vidal-Alaball J. Contextualising the 2019 E-Cigarette Health Scare: Insights from Twitter. Int J Environ Res Public Health. 2020;17(2236):1-10.

17. Ahmed W, Vidal-Alaball J, Downing J,López Seguí F. COVID-19 and the 5G Conspiracy Theory: Social Network Analysis of Twitter Data. J Med Internet Res. 2020;22(5):e19458

18. Tsao SF, Chen H, Tisseverasinghe T, Yang Y, Li L, Butt ZA. What social media told us in the time of COVID-19: a scoping review. Lancet Digit Health. 2021 Mar;3(3):e175-e194. doi: 10.1016/S2589-7500(20)30315-0. Epub 2021 Jan 28. PMID: 33518503; PMCID: PMC7906737. 